\title{
Taste Preference Following Cross-Innervation of Rat Fungiform Taste Buds
}

\author{
BRUCE OAKLEY \\ Department of Zoology, The University of Michigan, Ann Arbor, Michigan 48104
}

(Received 8 May 1969)

\begin{abstract}
OAKLeY, B. Taste preference following cross-innervation of rat fungiform taste buds. Physiol. BeHav. 4 (6) 929-933, 1969. It has been previously demonstrated that cross-innervation of rat taste buds alters some features of the taste input signal to the brain. In the present study the behavioral consequences of these alterations were assessed in a choice situation in which both water and taste solutions were available (two-bottle preference test). The taste preferences and aversions of operated control rats (one remaining IXth or chorda tympani nerve) were compared with those of experimental rats (one cross-regenerated IXth nerve). The reduced $\mathrm{NaCl}$ and sucrose preference observed 4 mo. postoperatively was probably a consequence of the specific effects of cross-innervation and not due simply to partial denervation. Little effect was observed at $8 \mathrm{mo}$. even though up to half of the existing tongue taste buds were cross-innervated.
\end{abstract}

Taste Taste preference Gustation Cross-innervation Sensory coding

THE CHORDA tympani and IXth nerves innervate taste buds in the anterior and posterior portions of the rat tongue, respectively [10]. A comparison of the summated neural responses from these two nerves indicates that the taste buds in the anterior and posterior tongue regions differ in their relative responsiveness to taste solutions $[8,10,13,16]$. Furthermore, both electrical and taste activation of these nerves indicate that they terminate in well-defined, partially overlapping regions in the nucleus fasiculus solitarius in the medulla $[2,5]$. Although the projections of these nerves to the medulla have been mapped out, no one has specifically mapped out the detailed projection of the tongue surface itself using adequate sensory stimulation (temperature, touch or taste). Nevertheless, the medullary projections for the whole chorda tympani and IXth nerves do suggest some degree of topographic representation for taste.

When taste buds are cross-innervated (e.g. when the IXth nerve is forced to innervate fungiform papillae on the anterior part of the tongue), the cross-regenerated nerve is changed in its relative responsiveness to different taste stimuli; that is, it responds like the nerve which normally innervates that tongue region [9]. For example, a cross-regenerated IXth nerve responds like the chorda tympani nerve which normally innervates the front of the tongue (e.g. with a relatively good response to $\mathrm{NaCl}$ ). Thus, cross-innervation should not only mis-wire the brain from the standpoint of the usual spatial correspondence between the anterior tongue and the anteriorlateral portion of the nu. fasiculus solitarius, but also in all likelihood should make the relative "signal strength" for different chemicals inappropriate as well. This experiment, then, represents an attempt to distort the taste information going into the brain and from the behavioral experiments on taste preferences and aversions make inferences about the process of coding of this information. The two-bottle preference test was used because it is a simple and reliable index of changes in the preferred or aversive qualities of chemical solutions.

\section{METHOD}

\section{A. Physiological}

Sixteen female Sprague-Dawley albino rats with an average initial weight of $240 \mathrm{~g}$ were used in this experiment. The operations upon the rats (Nembutal anesthesia: $50 \mathrm{mg} / \mathrm{kg}$ body weight) involved the three tongue sensory nerves: the lingual nerve (containing few if any taste fibers but other sensory fibers from the anterior part of the tongue), the chorda tympani nerve (composed of taste and other sensory fibers from the anterior tongue) and the IXth or glossopharyngeal nerve (composed of taste and other sensory fibers from the posterior tongue). The vagus nerve, which is presumed to innervate some taste buds in the epiglottis, pharynx and larynx was not disturbed except when it was necessary to cut a fine anastomosis that sometimes occurred between the laryngeal branch of the vagus and the IXth nerve.

Three types of taste nerve operations were performed, one experimental and two control:

$\mathrm{IX}_{\mathrm{c}}-\mathrm{C}_{\mathrm{p}}(\mathrm{N}=4)$ : In the experimental rats the central part of the IXth nerve was joined to the peripheral part of the chorda tympani such that the IXth nerve fibers regenerated into the anterior part of the tongue. During this operation the central portion of the right chorda tympani was avulsed

${ }^{1}$ The author would like to thank C. Bradley for technical assistance. Supported in part by U.S. Public Health Service Grant No. NB07072 and NSF Institutional (University of Michigan) Grant No. 109 to the author and by grants from the U.S. Public Health Service, National Science Foundation, Office of Naval Research, and Air Force Office of Scientific Research to T. H. Bullock. 
and the right lingual nerve was cut. In addition a week before behavioral testing the left IXth nerve and the left chorda tympani were cut in a second operation, which for 2 experimental rats occurred after 4 months following nerve crossing, and for 2 others after 8 months. (Two additional experimental animals died before behavioral testing during the second operation.)

IX-norm $(N=5)$ : In this control group all of the tongue sensory nerves (IX, chorda tympani, lingual) were cut 1 week before behavioral testing, except for the left lingual and right IXth nerve.

Ch-norm $(\mathrm{N}=5)$ : In this control group all tongue sensory nerves were cut 1 week before behavioral testing, except for the left lingual and the right chorda tympani.

Thus, the design of the experiment eliminated all of the tongue taste input save that provided by the cross-regenerated IXth nerve $\left(I X_{c}-C_{p}\right)$ in the experimental groups, and either a single IXth or single chorda tympani nerve in the 2 control groups. (As predicted on the basis of previous attempts to eliminate taste preference by peripheral denervation $[1$, $12,14,15]$, the control groups were not appreciably different from normal rats.) Additional information on operative procedure can be found elsewhere [9].

\section{B. Behavioral Testing}

For the purposes of behavioral testing the rats were divided into two groups. The rats in the 4 mo. Group ( $2 \mathrm{IX}_{\mathrm{c}}-\mathrm{C}_{\mathrm{p}}$, 3 IX-norm, 2 Ch-norm) were tested with sodium chloride, sucrose and quinine hydrochloride. These tests required two months to complete and were started 4 months after the nervecrossing in the experimental rats. Because the changes in preference and aversion toward taste solutions were not dramatic, all rats in the second or $8 \mathrm{mo}$. Group were pretested just prior to the second operation for the experimental rats and just prior to the single operation on the control rats. Thus, for the $8 \mathrm{mo}$. Group taste behavior was assessed eight months after nerve crossing just before and after elimination of unwanted taste nerves from the tongue of the experimental and control rats. The rats in the 8 mo. Group $\left(2 \mathrm{IX}_{\mathrm{c}}-\mathrm{C}_{\mathrm{p}}\right.$, 2 IX-norm, 3 Ch-norm) were tested with sodium chloride, sucrose and sodium saccharin. Repeated tests and additional taste solutions were not used because it was felt that some unwanted reinnervation of the tongue might occur in about 2 months after cutting the nerves. The histological results from 5 control rats that were not sacrificed for histology for up to three and one half months after taste nerve sectioning indicated some nerve regrowth.

In the actual testing procedure (two-bottle preference test) the rats were housed in individual cages and given food ad libitum. On the front of each cage were 2 inverted graduated cylinders, one containing water and the other some concentration of the taste solution being tested. The concentration was changed every $48 \mathrm{hr}$, and every $24 \mathrm{hr}$ the left-right position of the water and taste solutions interchanged and the volume intake recorded for the water and the taste solution. All chemicals, with the exception of quinine were presented in an ascending series of approximately one-third molar concentration steps, i.e., $\mathrm{NaCl}(0.01 \mathrm{M}, 0.02 \mathrm{M}, 0.05 \mathrm{M}, 0.1 \mathrm{M}$, . . 1.M), sucrose $(0.01 \mathrm{M}, 0.02 \mathrm{M}, \ldots .2 .0 \mathrm{M})$, Na saccharin $(0.0002 \mathrm{M}, 0.0005 \mathrm{M}, \ldots .0 .01 \mathrm{M})$. QHCl (quinine monohydrochloride hydrate) was presented in a descending series of concentration steps $(0.0001 \mathrm{M}, 0.00005 \mathrm{M}, 0.00002 \mathrm{M}, \ldots)$ until no longer discriminated from water. It required about 3 weeks, to test the preference or aversion toward a given chemical. The detailed testing procedure has been outlined in an earlier paper [11].

\section{Histology}

Five control rats were sacrificed about three and one half months after partial denervation of their tongues. The other control rats and the experimental rats were sacrificed immediately after behavioral testing which required about 2 months to complete. The tongues were fixed in 10 per cent formalin and embedded in paraffin. Complete $10 \%$ serial, horizontal sections of the foliate and circumvallate papillae were mounted on slides and stained with Heidenhain's iron haematoxylin. This stain leaves a small dark spot around the taste pore which facilitated the task of counting numerous taste buds in serial sections [4]. In each rat the first 50 sections of the tip of the anterior part of the tongue were discarded and then the next 325 serial sections were examined for fungiform taste buds. An examination of 10 normal rats indicated that an average of 47 per cent of the fungiform papillae are located in this $3.25 \mathrm{~mm}$ block of tissue $0.5-3.75 \mathrm{~mm}$ from the tip of the tongue.

RESULTS

\section{A. Behavior}

All control rats behaved as if they were normal animals with regard to their preference and aversion toward the various taste solutions. Among the experimental animals there were no complete eliminations or inversions of taste preference or aversion following cross-innervation of the fungiform taste buds. The experimental animals of the 4 mo. Group (No. 2 and No. 4) did display a reduced volume intake of sodium chloride solutions and a peak volume intake at $0.5 \mathrm{M}$, which constituted 76 per cent of their total fluid intake (Fig. 1). $0.5 \mathrm{M} \mathrm{NaCl}$ is reliably avoided by normal rats and was avoided by the experimental animals in the 8 mo. Group (No.

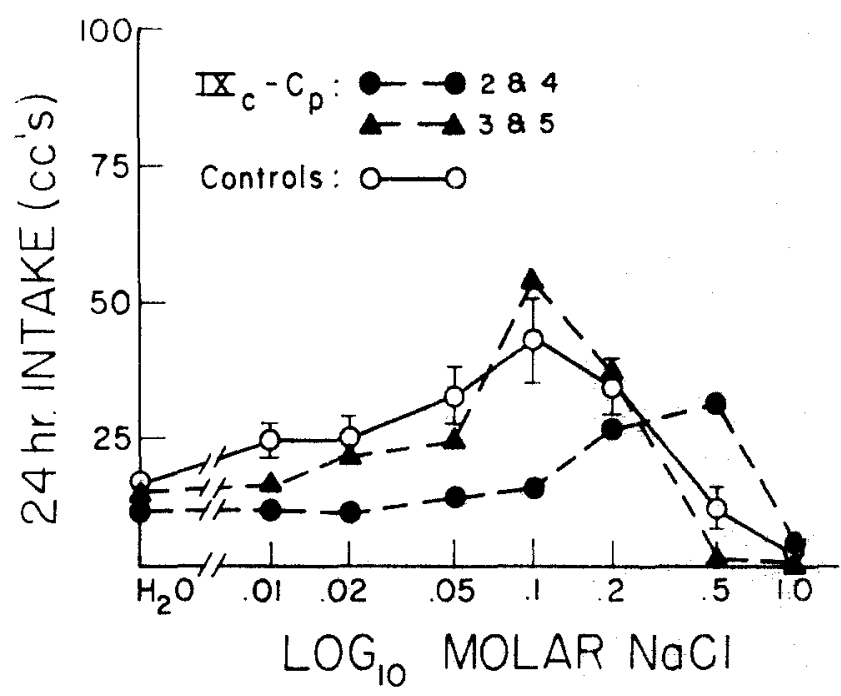

FIG. 1. Daily intake of sodium chloride as a function of concentration. Average curves for the $4 \mathrm{mo}$. Group experimental rats (\#2 and \#4) and the 8 mo. Group experimental rats ( $\# 3$ and \#5). The curve for the operated control rats is an average of data from ten rats and is plotted \pm one S.E. 


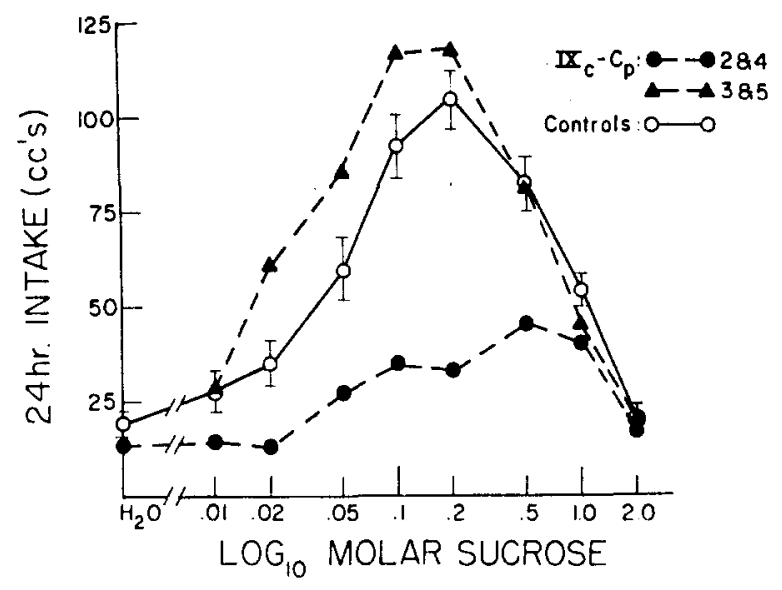

FIG. 2. The same as Fig. 1, but for sucrose intake.

3 and No. 5), whose intake curves were indistinguishable from those of the ten operated control animals. A similar reduction in preference for the $4 \mathrm{mo}$. experimental animals, but not the 8 mo. Group, occurred with sucrose (Fig. 2). The only known differences between the treatment of the 2 Groups of experimental animals was in the length of time allowed for adjustment to cross-innervation and in the pre-test given to the 8 mo. Group before the unwanted tongue nerves were cut. As will be evident from the histological results there were no differences apparent in the number or distribution of remaining taste buds, and therefore in unwanted reinnervation of the tongue.

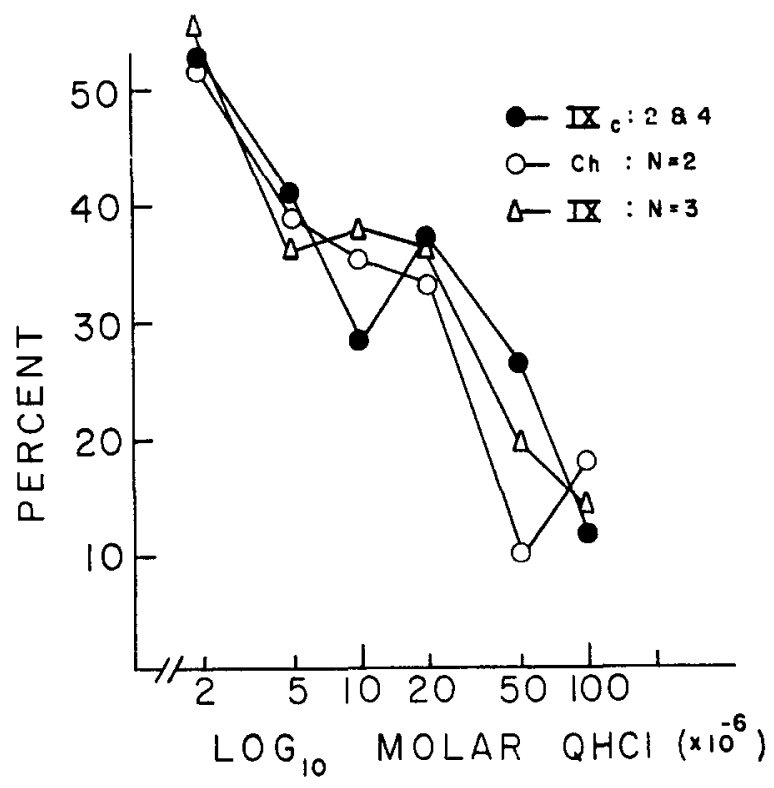

FIG. 3. Quinine preference. The $y$ axis is the intake from the tube containing quinine as a percentage of the total fluid intake: (cc $\left.\mathrm{QHCl} / \mathrm{cc} \mathrm{QHCl}+\mathrm{cc}_{2} \mathrm{O}\right) \times 100$. Filled circles: cross-regenerated IXth nerve rats in the 4 mo. Group. Open circles: two operated control rats with a single chorda tympani nerve intact. Open triangles: three operated control rats with a single IXth nerve intact.

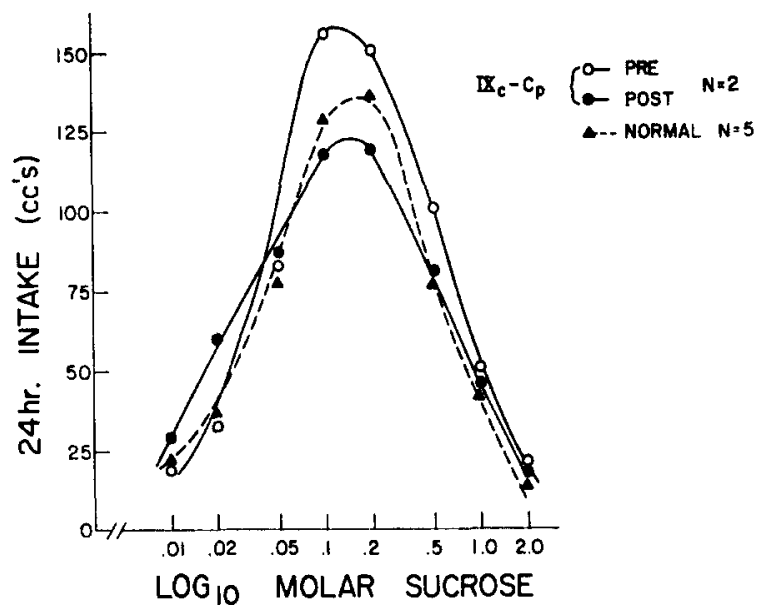

FIG. 4. Average daily intake of sucrose as a function of concentration. The two experimental rats $(8 \mathrm{mo}$. Group) were tested before and after removal of unwanted tongue sensory nerves The normal values are those of the 5 control rats tested before removal of the unwanted tongue sensory nerves.

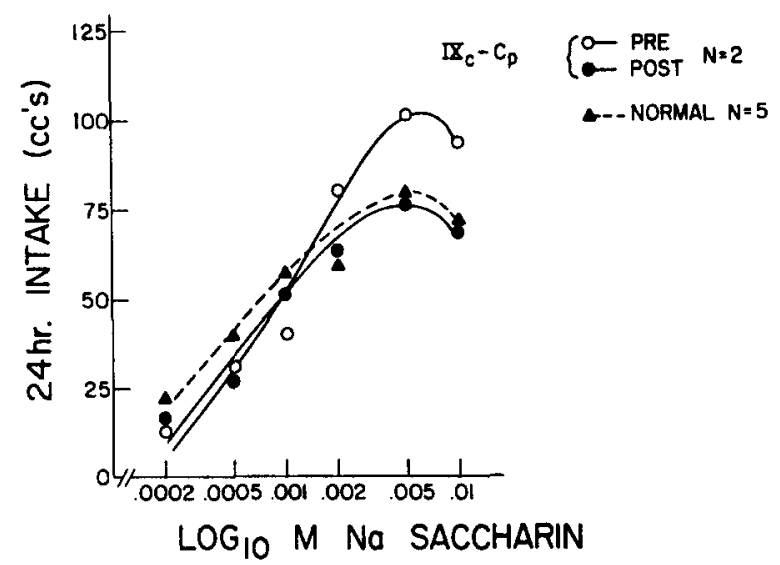

FIG. 5. The same as Fig. 4, but for sodium saccharin intake.

Aversion to quinine in the 4 mo. Group was less clearly affected by nerve crossing (Fig. 3), although the experimental animals did have a higher rejection threshold (the quinine concentration at which 25 per cent of the fluid intake is quinine) than the controls. It would appear, on the basis of Figs. 1-3, that preference was more severely impaired than aversion in the 4 mo. Group. As demonstrated by the intake curves for sucrose and $\mathrm{Na}$ saccharin before and after cutting unwanted tongue sensory nerves (Figs. 4 and 5) the preference behavior of the experimental animals in the 8 mo. Group was little affected by nerve crossing. This is indicated also by the following three quantitative measures, derived from Figs. 4 and 5 , of the stability of the sucrose and saccharin intake:

1. $X$ axis translation: The most preferred concentration (peak preference point) shifted less than $0.1 \log$ unit.

2. $Y$ axis translation: There was no more than a 25 per cent decrease in average volume intake of the three most preferred concentrations. 
3. Area under the increasing preference (rising) portion of the intake curves: There was a 5 per cent loss in area for sucrose and a 15 per cent loss for Na saccharin. This area reflects the extent to which taste stimulates drinking in excess of normal daily water intake.

\section{B. Histology}

All tongues were serially sectioned and examined for taste buds in the fungiform, foliate and circumvallate papillae. The ideal situation in the experimental rats would have been to have had a full complement of taste buds in the fungiform papillae on the right side and no taste buds elsewhere on the tongue. Table 1 shows the actual distribution of taste buds in the experimental rats. The role of the cross-regenerated IXth nerve in providing taste input is estimated in the last column. Thus, in rats No. 3 and No. 5 only about 15 per cent of the taste buds remained on the tongue (normally about 1000 taste buds) and about one half of those remaining were cross-innervated. Clearly, there was little unwanted or spurious regeneration and the goal of making the crossinnervated taste buds an important source of taste input from the tongue appears to have been achieved. Yet, it was these two experimental rats that showed quite normal preferenceaversion behavior. In several of the control rats unwanted regeneration of cut tongue nerves did occur, particularly in those which were not sacrificed until two months after completion of behavioral testing.

TABLE 1.

Taste Bud Distribution ${ }^{1}$

\begin{tabular}{|c|c|c|c|c|}
\hline Rat & Fung. & Foliate & Vallate & $\% \mathbf{I X}$ \\
\hline \multirow{2}{*}{$\not 2$} & 7 & 4 & \multirow[b]{2}{*}{95} & \multirow{2}{*}{$22 \%$} \\
\hline & 62 & 110 & & \\
\hline \multirow{2}{*}{$\# 4$} & 9 & N.A. & \multirow[b]{2}{*}{ N.A. } & \multirow[b]{2}{*}{--} \\
\hline & 76 & & & \\
\hline \multirow{2}{*}{$\not 3$} & 2 & 32 & \multirow[b]{2}{*}{41} & \multirow{2}{*}{$45 \%$} \\
\hline & 66 & 5 & & \\
\hline \multirow{2}{*}{$\not 5$} & 16 & 0 & \multirow{2}{*}{13} & \multirow{2}{*}{$48 \%$} \\
\hline & 69 & 46 & & \\
\hline
\end{tabular}

1The location and number of taste buds on the tongues of the four experimental rats. Left and right have not been given for the circumvallate papilla which lies on the midline of the tongue. The last column represents the percentage of the total number of taste buds on the tongue which were definitely innervated by the crossregenerated IXth nerve, namely the right fungiform taste buds. It is possible that some or all of the right foliate and circumvallate taste buds were also innervated by the cross-regenerated IXth nerve. $\mathrm{NA}=$ not available.

\section{DISCUSSION}

It isn't possible at present to specify what physiological changes in taste input occur as a result of cross-innervation.
For example, even if one knew the number of taste fibers in the IXth and chorda tympani nerves, there is no basis for assuming that all taste fibers have identical or equivalent roles in controlling drinking in preference behavior. Nor do we know the effects of cross-innervation upon the chemical specificity of individual taste fibers. Nevertheless, it is likely, in view of previous electrophysiological recording from cross-regenerated nerves [ 9 ], that at the least the relative stimulating effectiveness of different chemicals (e.g. $\mathrm{NaCl} v s$. sucrose) has been substantially altered in comparison with normal IXth nerve input.

Cross-innervation of the right fungiform taste buds resulted in a reduced preference for $\mathrm{NaCl}$ and sucrose in the $4 \mathrm{mo}$. Group. (It is impossible to account for this depressed preference on the basis of fewer taste buds. Rat No. 2 actually had more tongue taste buds than either rats No. 3 or No. 5 , whose behaviors were near normal.) The effect upon $\mathrm{NaCl}$ intake was greater than that reported by Pfaffmann [12], who bilaterally transected the chorda and IXth nerves, and by Vance [15] who cut the chorda, IXth and pharyngeal branch of the $\mathrm{Xth}$ nerve. Thus, the disturbed $\mathrm{NaCl}$ preference observed in the present experiment was quite possibly due to the specific influence of mis-wiring by cross-innervation. If these cross-connections had, instead, been behaviorally ineffective, then the deficits should have been no more severe than those observed by Pfaffmann and Vance in the absence of the chorda tympani and both IXth nerves.

In considering the role of learning experiences with taste stimuli which could account for the difference between the two Groups of experimental rats it is worthwhile pointing out that the $4 \mathrm{mo}$. Group rats were unresponsive to sucrose even after three weeks of experience with drinking $\mathrm{NaCl}$. Nevertheless, some adaptive adjustment to the disturbances resulting from cross-innervation, including the respecification of central taste connections, cannot be ruled out for the 8 mo. Group.

In the face of stable preference behavior one must also ask whether there are some dominant non-lingual sources of taste input, or whether taste is even essential for controlling this kind of drinking behavior. There is evidence supporting the relevance of taste input in the control of preference behavior. For example, in the absence of taste stimulation two bottle preference behavior deteriorates and fails as in cases where the taste relays have been destroyed in the medulla or thalamus $[2,11]$ and in experiments where the oral areas are bypassed by self-delivery of solutions directly into the stomach [3]. Recently, Mook and Kozub [7] have concluded that taste plays no role in controlling $24 \mathrm{hr}$ intake in a non-deprived rat that drinks from a single bottle of saline, although they do believe taste is important in choice situations. However, there is no sound basis for their assumption that water tubed into the rat's stomach is a neutral condition which would permit the neural consequences of saline taste to be freely expressed as drinking behavior. On the contrary it would be most surprising if rats would continue to lick saline when it results (by tubing water into the stomach) in increasing overhydration and osmotic imbalance. The homeostatic requirement for water balance is likely to predominate over taste stimulation. If saline drinking is not punished by overhydration, then taste may even be as important in controlling ingesting of saline from a single bottle as it is in the two bottle situation.

Regarding non-lingual taste buds elsewhere in the oral cavity, it is possible that these taste buds simply outnumbered 
and overwhelmed the abnormal input from the cross-innervated fungiform taste buds. Although taste buds in the roof of the mouth have been described [6] there is no complete study of the distribution or number of all non-lingual taste buds. Given both that preference behavior was only partially eliminated (reduced) and the general inability of other investigators to eliminate preference behavior by peripheral denervation of the tongue $[1,12,14,15]$, it is appropriate to determine the distribution and number of non-lingual taste buds in the mouth and pharynx of the rat.

If one can temper the influence of (a) physiological adjustment to cross-innervation (e.g. changed connections in the medulla) and/or (b) masking of crossed input by a large non-lingual taste input, then behavior in preference and aversion may be even more dramatically affected by cross-innervation of taste buds.

\section{REFERENCES}

1. Akaike, N., Y. Hiji and K. Yamada. Taste preference and aversion in rats following denervation of the chorda tympani and IXth nerve. Kumamoto med. J. 18: 108-109, 1965.

2. Blomquist, A. J. and A. Antem. Localization of the terminals of the tongue afferents in the nucleus of the solitary tract. J. comp. Neurol. 124: 127-130, 1965.

3. Borer, Katarina T. Disappearance of preferences and aversions for sapid solutions in rats ingesting untasted fluids. J. comp. physiol. Psychol. 65: 213-221, 1968.

4. Guth, L. The effects of glossopharyngeal nerve transection on the circumvallate papilla of the rat. Anat. Rec. 128: 715-732, 1957.

5. Halpern, B. P. and Linda M. Nelson. Bulbar gustatory responses to anterior and to posterior tongue stimulation in the rat. Am. J. Physiol. 209: 105-110, 1965.

6. Kaplick, M. Uber Vorkommen, Verteilung und histologische Beziehungen der Geschmacksknospen am Munddach einiger Säuger, besonders der Nagetiere. Z. Zellforsch. Mikroskop. Anat. 38: 571-590, 1953.

7. Mook, D. G. and F. J. Kozub. Control of sodium chloride intake in the non-deprived rat. J. comp. physiol. Psychol. 66: 105-109, 1968.

8. Oakley, B. Altered taste responses from cross-regenerated taste nerves in the rat. In: Olfaction and Taste II, edited by $\mathrm{T}$. Hayashi. Oxford: Pergamon Press, 1967, pp. 535-548.
9. Oakley, B. Altered temperature and taste responses from crossregenerated sensory nerves in the rat's tongue. J. Physiol. 188: 353-371, 1967.

10. Oakley, B. and R. M. Benjamin. Neural mechanisms of taste. Physiol. Rev. 46: 173-211, 1966.

11. Oakley, B. and C. Pfaffmann. Electrophysiologically monitored lesions in the gustatory thalamic relay of the albino rat. $J$. comp. physiol. Psychol. 55: 155-160, 1962.

12. Pfaffmann, C. Taste preference and aversion following lingual denervation. J. comp. physiol. Psychol. 45: 393-400, 1952.

13. Pfaffmann, C., G. L. Fisher and M. K. Frank. The sensory and behavioral factors in taste preferences. In: Olfaction and Taste II, edited by T. Hayashi. Oxford: Pergamon Press, 1967, pp. 361-382.

14. Richter, C. P. Salt appetite of mammals: its dependence on instinct and metabolism. In: L'instinct dans le comportment des animaux et de l'homme. Paris: Masson et Cie, 1956, pp. $577-632$.

15. Vance, W. B. Hypogeusia and taste preference behavior in the rat. Life Sci. 6: 743-748, 1967.

16. Yamada, K. Gustatory and thermal responses in the glossopharyngeal nerve of the rat. Jap. J. Physiol. 16: 599-611, 1966. 